\title{
BTF Image Space Utmost Compression and Modelling Method
}

\author{
Michal Haindl and Jiří Filip \\ Institute of Information Theory and Automation \\ Academy of Sciences CR, 18208 Prague, Czech Republic \\ \{haindl,havlicek\}@utia.cas.cz
}

\author{
Michael Arnold \\ DaimlerChrysler AG \\ 71059 Sindelfingen, Germany
}

\begin{abstract}
The bidirectional texture function (BTF) describes texture appearance variations due to varying illumination and viewing conditions. This function is acquired by large number of measurements for all possible combinations of illumination and viewing positions hence some compressed representation of these huge BTF texture data spaces is obviously inevitable. In this paper we present a novel efficient probabilistic model-based method for multispectral BTF texture compression which simultaneously allows its efficient modelling. This representation model is capable of seamless BTF space enlargement and direct implementation inside the graphical card processing unit. The analytical step of the algorithm starts with BTF texture surface estimation followed by the spatial factorization of an input multispectral texture image. Single band-limited factors are independently modelled by their dedicated $3 D$ causal autoregressive models $(C A R)$. We estimate an optimal contextual neighbourhood and parameters for each CAR. Finally the synthesized multiresolution multispectral texture pyramid is collapsed into the required size fine resolution synthetic smooth texture. Resulting BTF is combined in a displacement map filter of the rendering hardware using both multispectral and range information, respectively. The presented model offers immense BTF texture compression ratio which cannot be achieved by any other sampling-based BTF texture synthesis method.
\end{abstract}

\section{Introduction}

A physically correct virtual models visualization cannot be accomplished without nature-like colour textures covering virtual or augmented reality scene objects. These textures can be either smooth or rough (also referred as the bidirectional texture function - BTF). The rough textures which have rugged surfaces do not obey the Lambert law and their reflectance is illumination and view angle dependent. Both types of textures occur in virtual scenes models can be either digitised natural textures or textures synthesised from an appropriate mathematical model. The former simplistic option suffers among others with extreme memory requirements for storage of a large number of digitised cross-sectioned slices through different material samples. Sampling solution become unmanageable for rough textures which require to store thousands of different illumination and view angle samples for every texture. Such a simple VR scene requires to store tera bytes of texture data which is far out of limits for any current real time hardware.

Several intelligent sampling methods [3], [4], [10], were proposed with the aim to diminish these huge memory requirements. All these methods are based on some sort of original small texture sampling and the best of them produce very realistic synthetic textures. However these methods require to store thousands images for every combination of viewing and illumination angle of the original target texture sample, they often produce visible seams, some of them are computationally demanding and they cannot generate textures unseen (unstored) by the algorithm.

Synthetic textures based on adaptive spatial models are far more flexible, extremely compressed (few tens of parameters to be stored only), they may be evaluated directly in procedural form, can be designed to meet certain constraints or properties, and can be used to seamlessly fill an infinite texture space. Mathematical models can only approximate real measurements which results in visual quality compromise of some methods. Several smooth texture modelling approaches, mostly monospectral but some also multispectral, were published, e.g., [2], [8], [1] and some survey articles are available [6] as well.

CAR type of random fields are appropriate for texture modelling not only because they do not suffer with some problems of alternative options (see [?], for details) but they are also easy to synthesise and still flexible enough to imitate a large set of natural and artificial textures. While the random field based models quite successfully represent high frequencies present in natural textures low frequencies are much more difficult for them. One possibility how to overcome this drawback is to use a multiscale random field 
model. Unfortunately CAR random fields, similarly as the majority of other Markovian types of random field models, are not invariant to multiple resolution decomposition (MRD) even for simple MRD like subsampling (they generally lose their Markovianity property) and become ARMA random fields instead. Fortunately for the representation and modelling applications we can avoid computationally demanding approximations of an ARMA multigrid random field by an infinite order (i.e., high order in practice) CAR random fields because there is no need to transfer information between single spatial factors hence it is sufficient to analyse and synthesise each resolution component independently.

\section{BTF Model}

We propose a novel BTF representation Fig.1 which combines an estimated range map parameters with parameters of a set of mutually coupled simultaneous causal autoregressive random (CAR) models. The multiscale CAR random field based models represent the smooth texture part of the data. This BTF representation allows BTF space enlargement to any required size and efficient easy simulation even inside a graphical processing unit.

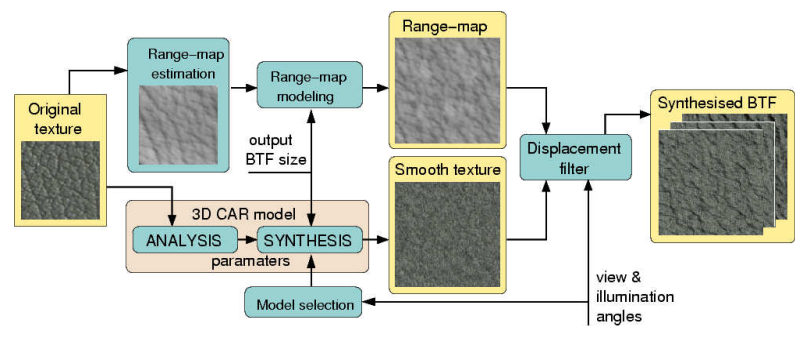

Figure 1. The overall BTF algorithm scheme.

The texture visual appearance during changes of viewing and illumination conditions is simulated using the displacement mapping technique (or approximated through the bump map filter). The obvious advantage of this solution is the possibility to use hardware support of displacement (bump) map technique in contemporary visualisation hardware.

The overall roughness of a textured surface significantly influences a BTF texture appearance. Such a surface can be specified using its range map, which can be estimated by several existing approaches, e.g., direct measurement, photometric stereo, shape from shading. The range map estimation within this paper was performed by shape from shading algorithm [5]. This method exploits the fact that image intensity $Y_{r}$ of each pixel observed in texture image is given according to Lambertian (in our implementation) reflectance map $Y_{r}=R\left(z_{r_{1}}, z_{r_{2}}, \theta, \mathbf{C}, \rho\right)$ :

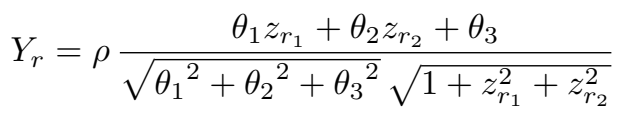

where $z_{r_{1}}$ and $z_{r_{2}}$ are surface slopes in directions $r_{1}$ and $r_{2}$, $\theta$ is vector from surface to illumination source, $\mathbf{C}$ is vector from surface to the camera and $\rho$ is albedo of observed material. The algorithm ignores multiple reflections, assumes known vectors $\mathbf{C}, \theta$, spatially invariant reflectance map and includes constrain which enforces integrability of surface slopes:

$$
\frac{\partial^{2} Z_{r}}{\partial r_{1} \partial r_{2}}=\frac{\partial^{2} Z_{r}}{\partial r_{2} \partial r_{1}}
$$

where $Z_{r}$ is the unknown surface height in the location $r=\left(r_{1}, r_{2}\right)$. This integrability condition is performed in the Fourier coefficient representation. The Lambertian reflectance assumption may deteriorate the range map estimation for some non-Lambertian texture surfaces, although results in [5] demonstrate still acceptable estimates even in these cases. Estimated range map is finally modelled using the simplified (i.e. 2D) CAR model analogously to the spectral information.

Input multispectral image is decomposed into a multiresolution grid and each resolution data factor is independently modelled by its dedicated CAR model. Each one generates a single spatial frequency band of the texture. An analysed texture is decomposed into multiple resolutions factors using Laplacian pyramid and the intermediary Gaussian pyramid $\ddot{Y}_{\bullet, i}^{(k)}$ which is a sequence of images in which each one is a low-pass down-sampled version of its predecessor where the weighting function (FIR generating kernel) $w$ is chosen subject to execute separability, normalization, symmetry and equal contribution constrains. The Gaussian pyramid for a reduction factor $n$ is

$$
\ddot{Y}_{r, i}^{(k)}=\downarrow_{r}^{n}\left(\ddot{Y}_{\bullet, i}^{(k-1)} \otimes w\right) k=1,2, \ldots,
$$

where $\ddot{Y}_{\bullet, i}^{(0)}=\bar{Y}_{\bullet, i}, \downarrow^{n}$ denotes down-sampling with reduction factor $n$ and $\otimes$ is the convolution operation.

$$
\ddot{Y}_{r}^{(k)}=\sum_{i, j=-l}^{l} \hat{w}_{i} \hat{w}_{j} \ddot{Y}_{2 r+(i, j)}^{(k-1)} .
$$

The Laplacian pyramid $\dot{Y}_{r, i}^{(k)}$ contains band-pass components and provides a good approximation to the Laplacian of the Gaussian kernel. It can be constructed by differencing single Gaussian pyramid layers:

$$
\dot{Y}_{r, i}^{(k)}=\ddot{Y}_{r, i}^{(k)}-\uparrow_{r}^{n}\left(\ddot{Y}_{\bullet, i}^{(k+1)}\right) \quad k=0,1, \ldots,
$$

where $\uparrow^{n}$ is the up-sampling with an expanding factor $n$. 


\subsection{CAR Factor Model}

Modelling general multispectral (e.g. colour) textures require three dimensional models to allow unrestricted spatial-spectral correlation representation. The advantage of 3D CAR models is that they can be solved, under several additional and acceptable assumptions, analytically. Multispectral textures are decomposed into a multi-resolution grid and each resolution data are independently modeled by their dedicated independent Gaussian noise driven CAR random field model. Let the digitized colour texture $Y$ is indexed on a finite rectangular three-dimensional $N \times M \times$ $d$ underlying lattice $I$, where $N \times M$ is the image size and $d$ is the number of spectral bands (i.e., $d=3$ for usual colour textures). Let us denote a simplified multiindex $r$ to have two components $r=\left[r_{1}, r_{2}\right]$. The first component is row and and the second one is column index, respectively. The (CAR) random field is a family of random variables with a joint probability density on the set of all possible realisations $Y$ of the $M \times N \times d$ lattice $I$, subject to the following condition:

$$
\begin{gathered}
p\left(Y \mid \gamma, \Sigma^{-1}\right)=(2 \pi)^{-\frac{d(M N-1)}{2}}\left|\Sigma^{-1}\right|^{\frac{(M N-1)}{2}} \\
\exp \left\{-\frac{1}{2} \operatorname{tr}\left\{\Sigma^{-1}\left(\begin{array}{c}
-I \\
\gamma^{T}
\end{array}\right)^{T} \tilde{V}_{M N-1}\left(\begin{array}{c}
-I \\
\gamma^{T}
\end{array}\right)\right\}\right\}, \\
\tilde{V}_{r-1}=\left(\begin{array}{cc}
\tilde{V}_{y y(r-1)} & \tilde{V}_{x y(r-1)}^{T} \\
\tilde{V}_{x y(r-1)} & \tilde{V}_{x x(r-1)}
\end{array}\right),
\end{gathered}
$$

where the used notion is: $\tilde{V}_{\delta \omega(r-1)}=\sum_{k=1}^{r-1} \Delta_{k} \Omega_{k}^{T}$. The 3D CAR model can be expressed as a stationary causal uncorrelated noise driven $3 \mathrm{D}$ autoregressive process:

$$
Y_{r}=\gamma X_{r}+e_{r}
$$

where $\gamma$ is the $d \times d \eta$ parameter matrix

$$
\gamma=\left[A_{1}, \ldots, A_{\eta}\right],
$$

$\eta=\operatorname{card}\left(I_{r}^{c}\right), \quad I_{r}^{c}$ is a causal neighbourhood, $e_{r}$ is a Gaussian white noise vector with zero mean and a constant but unknown covariance matrix $\Sigma$ and $X_{r}$ is a corresponding vector of $Y_{r-s}$ (design vector).

The selection of an appropriate CAR model support is important to obtain good modelling results. Too small contextual neighbourhood can not capture all details while inclusion of surplus neighbours add to the computational burden and can potentially degrade the performance of the model as an additional source of noise. The optimal neighbourhood can be found using the Bayesian decision rule for minimizing the average probability of decision error.
The most probable CAR model given past data, the normalWishart parameter prior and the uniform model prior is the model which maximise the statistics:

$$
\begin{aligned}
D_{j(r-1)} & =\frac{-d}{2} \ln \left|V_{x x(r-1)}\right|-\frac{\beta(r)-d \eta+d+1}{2} \ln \left|\lambda_{(r-1)}\right| \\
& +\frac{d^{2} \eta}{2} \ln \pi \sum_{i=1}^{d}\left[\ln \Gamma\left(\frac{\beta(r)-d \eta+d+2-i}{2}\right)\right. \\
& \left.-\ln \Gamma\left(\frac{\beta(0)-d \eta+d+2-i}{2}\right)\right]
\end{aligned}
$$

where $\beta(r)=\beta(0)+r-1, \beta(0)>1$, and

$$
\lambda_{(r)}=V_{y y(r)}-V_{x y(r)}^{T} V_{x x(r)}^{-1} V_{x y(r)} .
$$

Parameter estimations (6),(7) of the CAR model using the Bayesian method and the normal-Wishart parameter prior can be found analytically.

$$
\hat{\gamma}_{r-1}^{T}=V_{x x(r-1)}^{-1} V_{x y(r-1)}
$$

and

$$
\hat{\Sigma}_{r-1}=\frac{\lambda_{(r-1)}}{\beta(r)},
$$

where $V_{u z(r-1)}=\tilde{V}_{u z(r-1)}+V_{u z(0)}$ and matrices $V_{u z(0)}$ are the corresponding matrices from the normal-Wishart parameter prior. The estimates (5), (6),(7) can be also evaluated recursively if necessary.

The CAR model synthesis is very simple and the CAR random field can be directly generated from the model equation (3) using a multivariate Gaussian generator. The fineresolution synthetic texture is obtained from the pyramid collapse procedure.
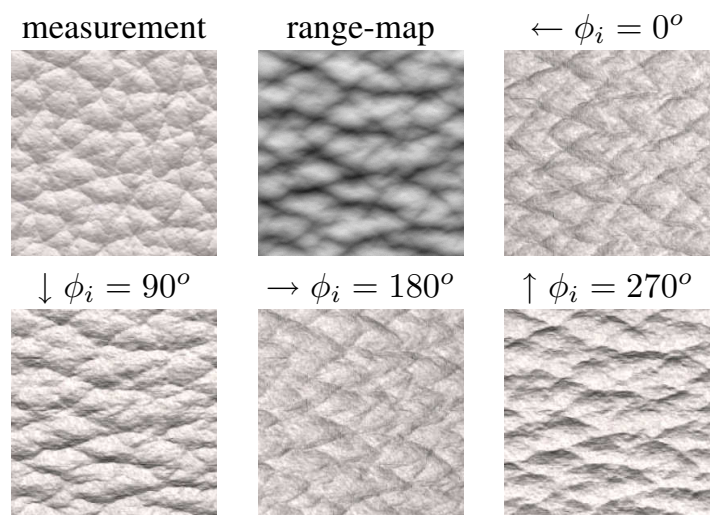

Figure 2. White leather examples and their BTF synthetic results for illumination angles $0^{\circ}, 90^{\circ}, 180^{\circ}$ and $270^{\circ}$. 


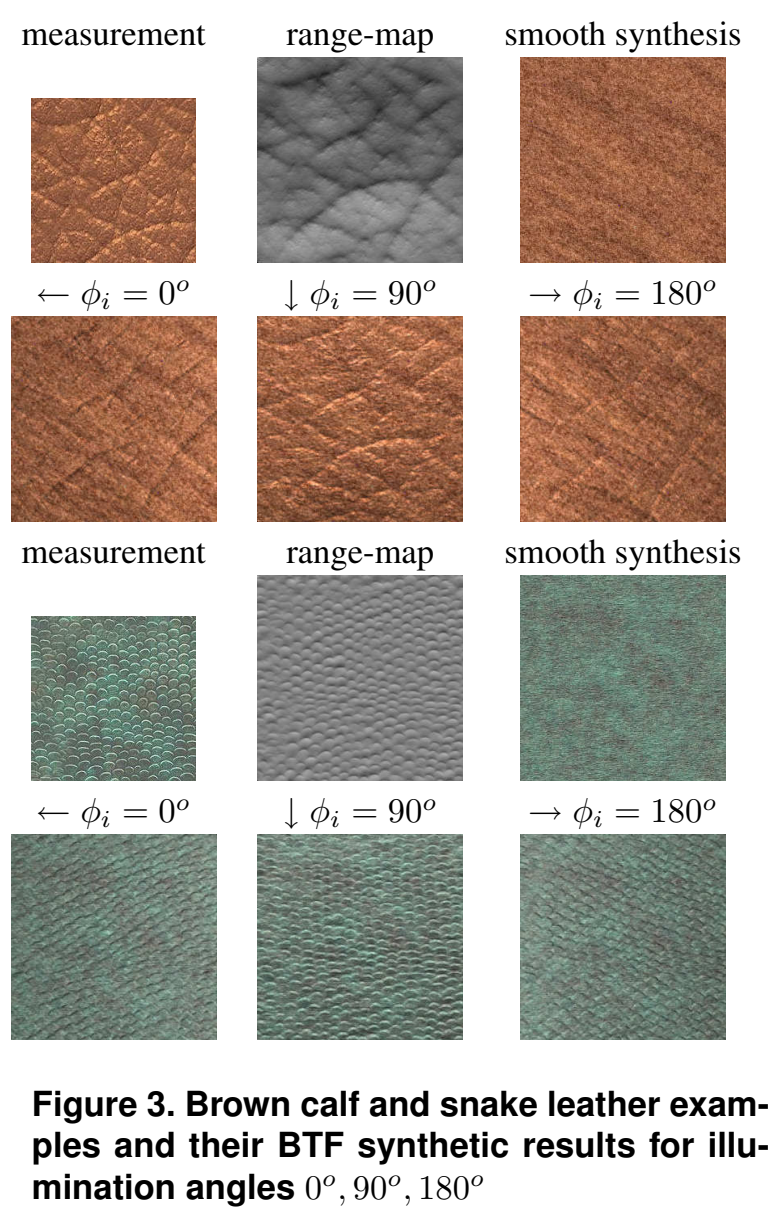

\section{Results}

We have tested the algorithm on several BTF colour texture databases - the University of Bonn [13] database, the Yale University [12]database and several our BTF measurements, such as corduroy, upholstery, carpet, knitwear or leather textures. The textures were modelled for several illumination directions and visually compared with their corresponding measurements. Three presented natural colour examples (Fig.2,Fig.3) illustrate the presented algorithm performance. Even if the leather textures (Fig.2) violates the CAR model stationarity assumption, the algorithm demonstrates its ability to model such BTF textures with very good visual realism. The displacement mapping technique algorithm option provides better results than the bump mapping alternative (not shown here). The multiscale models demonstrate [8] their clear superiority over their single-scale counterparts while the colour quality is comparable between single-scale and multi-scale alternative models.

\section{Summary and Conclusions}

Our test results prove extreme BTF data compression ratio of the proposed representation simultaneously with very realistic visual modelling quality. Some synthetic BTF textures reproduce given measured images so that both natural and synthetic texture are almost visually indiscernible and even the worst results can be used for preattentive BTF textures applications at least. The multi-scale approach is more robust and allows far better results than the singlescale one if the synthesis model is inadequate (lower order model, non stationary texture, etc.). Hence the CAR model is better suited for real time (e.g. using a graphical card processing unit) or web applications than most other published alternatives.

The presented method is based on the estimated model in contrast to prevailing intelligent sampling type of methods, and as such it can only approximate realism of the original measurement. However it offers unbeatable data compression ratio (tens of parameters per texture only), easy simulation of even non existing (previously not measured) BTF textures and fast seamless synthesis of any texture size.

\section{Acknowledgements}

This research was supported by the EC project no. IST2001-34744 RealReflect, grant No.A2075302 of the Grant Agency of the Academy of Sciences CR and partially by the grant MŠMT No. ME567 MIXMODE. The authors wish to thank R. Klein of the University of Bonn for providing us with the corduroy BTF measurements.

\section{References}

[1] J. Bennett and A. Khotanzad. Multispectral random field models for synthesis and analysis of color images. IEEE Trans. on Pattern Analysis and Machine Intelligence, 20(3):327-332, March 1998.

[2] J. Besag. Spatial interaction and the statistical analysis of lattice systems. Journal of the Royal Statistical Society, Series B, B-36(2):192-236, February 1974.

[3] A. A. Efros and T. K. Leung. Texture synthesis by nonparametric sampling. In ICCV (2), pages 1033-1038, 1999.

[4] R. Frankot and R. Chellappa. A method for enforcing integrability in shape from shading algorithms. IEEE Transactions on Image Processing, 10(4):439-451, July 1988.

[5] M. Haindl. Texture synthesis. CWI Quarterly, 4(4):305-331, December 1991.

[6] M. Haindl and V. Havlíček. Multiresolution colour texture synthesis. In K. Dobrovodský, editor, Proceedings of the 7th International Workshop on Robotics in Alpe-Adria-Danube Region, pages 297-302, Bratislava, June 1998. ASCO Art.

[7] D. Heeger and J. Bergen. Pyramid based texture analysis/synthesis. In ACM SIGGRAPH 95, pages 229-238. ACM Press, 1995. 
[8] M. Koudelka, S. Magda, P. Belhumeur, and D. Kriegman. Acquisition, compression, and synthesis of bidirectional texture functions. In Texture 2003: Proceedings of 3th International Workshop on Texture Analysis and Synthesis, pages 47-52. Heriot-Watt University, October 2003.

[9] M. Sattler, R. Sarlette, and R. Klein. Efficient and realistic visualization of cloth. In Eurographics Symposium on Rendering 2003, pages 167-178, June 2003. 